\title{
[ 392$]$
}

XL. On the Passage of Electricity through Gases exposed to Röntgen Rays. By J. J. Thomson, M.A., F.R.S., Cavendish Professor of Experimental Physics, Cambridge, and E. Rutherford, M.A., Trinity College, Cambridge, 1851 Exhibition Scholar, New Zealand University*.

THE facility with which a gas, by the application and 1 removal of Röntgen rays, can be changed from a conductor to an insulator makes the use of these rays a valuable means of studying the conduction of electricity through gases, and the study of the properties of gases when in the state into which they are thrown by the rays promises to lead to results of value in connexion with this subject. We have during the past few months made a series of experiments on the passage of electricity through gases exposed to the rays, the results of these experiments are contained in the following paper.

A gas retains its conducting property for a short time after the rays have ceased to pass through it. This can readily be shown by having a charged electrode shielded from the direct influence of these rays, which pass from the vacuum-tube through an aluminium window in a box covered with sheet lead; then, though there is no leak when the air in the neighbourhood of the electrode is still, yet on blowing across the space over the aluminium window on to the electrode the latter immediately begins to leak.

To make a more detailed examination of this point we used the following apparatus.

A closed aluminium ressel is placed in front of the window through which the rays pass. A tube through which air can be blown by a pair of bellows leads into this vessel : the rate at which the air passed through this tube was measured by a gas-meter placed in series with the tube; a plug of glass wool was placed in the tube leading to the vessel to keep out the dust. The air left the aluminium. vessel through another tube, at the end of which was placed the arrangement for measuring the rate of leakage of electricity (usually a wire charged to a high potential placed in the axis of an earthconnected metal tube through which the stream of gas passed, the wire being connected with one pair of quadrants of an electrometer). This arrangement was carefully shielded from the direct effect of the rays, and there was no leak unless a current of air was passing through the apparatus; when,

* Communicated by the Authore, having been read before Sectiun $A$ of the British Association, 1896. 
however, the current of air was flowing there was a considerable leak, showing that the air after exposure to the rays retained its conducting properties for the time (about $\frac{1}{2}$ second) it took to pass from the aluminium vessel to the charged electrode.

We tried whether the conductivity of the gas would be destroyed by heating the gas during its passage from the place where it was exposed to the rays to the place where its conductivity was tested. To do this we inserted a piece of porcelain tubing which was raised to a white heat ; the gas after coming through this tube was so hot that it could hardly be borne by the hand; the conductivity, however, did not seem to be at all impaired. If, however, the gas is made to bubble through water every trace of conductivity seems to disappear. The gas also lost its conductivity when forced through a plug of glass wool, though the rate of flow was kept the same as in an experiment which gave a rapid leak; if the same plug was inserted in the system of tubes before the gas reached the vessel where it was exposerl to the Röntgen rays, in this case the conductivity was not diminished. This experiment seems to show that the structure in virtue of wbich the gas conducts is of such a coarse character that it is not able to survive the passage through the fine pores in a plug of glass wool. A diaphragm of fine wire gauze or muslin does not seem to affect the conductivity.

A very suggestive result is the effect of passing a current of electricity through the gas on its way from the aluminium vessel where it is exposed to the Röntgen rays to the place where its conductivity is examined. We tested this by inserting a metal tube in the circuit, along the axis of which an insulated wire was fixed connected with one terminal of a battery of small storage-cells, the other terminal of this battery was connected with the metal tube ; thus as the gas passed through the tube a current of electricity was sent through it. The passage of a current from a few cells was sufficient to greatly diminish the conductivity of the gas passing through the tube, and by increasing the number of cells the conductivity of the gas could be entirely destroyed. Thus the peculiar state into which a gas is thrown by the Röntgen rays is destroyed when a current of electricity passes through it. It is the current which destroys this state, not the electric field; for if the central wire is enclosed in a glass tube so as to stop the current but maintain the electric field, the gas passes through with its conductivity unimpaired. The current produces the same effect on the gas as it would produce on a very weak solution of an electrolyte. For imagine such a solution 
to pass through the tubes instead of the gas ; then if enough electricity passed through the solution to decompose all the electrolyte the solution when it emerged would be a nonconductor; and this is precisely what happens in the case of the gas. We shall find that the analogy between a dilute solution of an electrolyte and gas exposed to the Röntgen rays holds through a wide range of phenomena, and we have found it of great use in explaining many of the characteristic properties of conduction through gases.

Thus Röntgen rays supply a means of communicating a charge of electricity to a gas. To do this, take an insulated wire charged up to a high potential and surrounded by a tube made of a non-conducting substance : let this tube lead into a large insulated metallic vessel connected with an electrometer. If now air which has been exposed to Röntgen rays is blown through the tube into this vessel the electrometer will be deflected. This proves that the gas inside the vessel is charged with electricity. If the Röntgen rays are stopped and the gas blown out of the vessel the charge disappears. In these experiments we took precautions against dust.

The fact that the passage of a current of electricity through a gas destroys its conductivity explains a very characteristic property of the leakage of electricity through gases exposed to Röntgen rays; that is, for a given intensity of radiation the current through the gas does not exceed a certain maximum value whatever the electromotive force may be, the current gets, as it were, "saturated." The relation between the electromotive force and the current is shown in the following curve, where the ordinates represent the current and

Fig. 1.

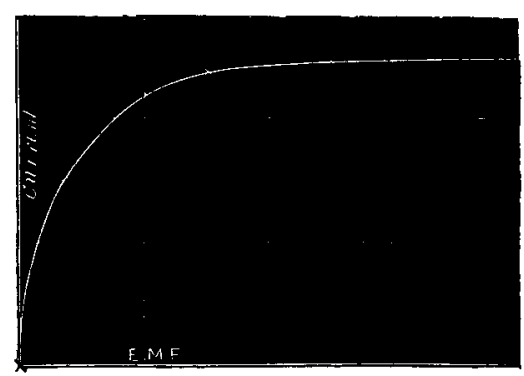

the abscissæ the electromotive force. It is evident that this saturation must occur if the current destroys the conducting power of the gas, and that the maximum current will be the current which destroys the conductivity at the $\operatorname{sam} \theta$ rate as 
of Electricity throngh Guses exposed to Röntgen Rays. 395

this property is produced by the Röntgen r.tys. If we regarl the gas as an electrolyte, then the passage of a quantity $e$ of olectricity will destroy $e / \epsilon$ of the conducting particles, wher $\epsilon$ is the charge carried by one of these particles. Lat $n$ be the number of conducting particles in unit volume of the gas, $q$ the rate at which these are produced by the rays, $\alpha n^{2}$ the rate at which these disappear independently of the passage of the current, $\iota$ the current through unit area of the gas, $l$ the distance between the elestrodes. Then we have

$$
\frac{d n}{d t}=q-\alpha n^{2}-\frac{\iota}{l \epsilon} ; . . . \quad . \quad . \quad .
$$

so that when the state of the gas is steady,

$$
0=q-\alpha n^{2}-\frac{\iota}{l \epsilon} \text {. . . . . . . . }
$$

When the current is small this equation gives

$$
n^{2}=q / a ;
$$

and as the number of conducting particles is independent of the current, the current will be proportional to the E.M.F. This corresponds to the straight part of the curve.

In the general case the current is proportional to the product of $n$, the number of conducting molecules, and the potential gradient. If $\mathrm{E}$ is the difference of potential between the plates, $U$ the sum of the velocities of the positively and negatively electrified particles when the potential gradient is unity, we have

$$
\iota=n \epsilon \mathrm{UE} / l \text { or } n=\frac{l \iota}{\epsilon \mathrm{UE}} \text {. }
$$

Substituting this value of $n$ in equation (2), we get

$$
0=q-\frac{a l^{2} \iota^{2}}{\epsilon^{2} \mathrm{U}^{2} \mathrm{E}^{2}}-\frac{\iota}{l \epsilon} \text {. . . . . . . }
$$

We see from this that $\iota$ approaches the limit $q \varepsilon l$. Thus the limiting current is proportional to the distance between the electrodes ; so that when we approach saturation the current will increase as the distance between the electrodes increases, and we get what is at first sight the paradoxical result that a thin layer of air offers a greater resistance to the passage of a current than a thicker one. This is, however, easily accounted for if we remember that the current destroys the conductingpower, and that as in a thicker layer there are more conducting particles than in a thinner one the current required to destroy them all will be greater. 
The experiments show that the effect of the distance between the electrodes (two parallel plates) on the current is very marked. The following tables show the result of some experiments on this point.

Potential-Difference between Electrodes 60 volts.

\begin{tabular}{|c|c|c|c|}
\hline $\begin{array}{l}\text { Distance between e } \\
\text { in millimetr }\end{array}$ & $\begin{array}{l}\text { sctrodes, } \\
\text { s. }\end{array}$ & & $\begin{array}{r}\text { Current (arl } \\
\text { scale.) }\end{array}$ \\
\hline$\cdot 1$ & & & . . 9 \\
\hline$\cdot 12$ & . & . & 15 \\
\hline .25 & . & . & 21 \\
\hline$\cdot 5$ & . & . & 37 \\
\hline 1 &. & . & . . 50 \\
\hline $1 \cdot 5$ &. & . & . . 62 \\
\hline 3 & $\cdot \cdot$ & - & • $\quad \begin{array}{r}91 \\
110\end{array}$ \\
\hline & . . & & . .110 \\
\hline
\end{tabular}

With this large potential-difference the current was saturated in all the experiments.

The next table contains measurements with a small potentialdifference.

Potential-Difference between Electrodes 1.3 volt.

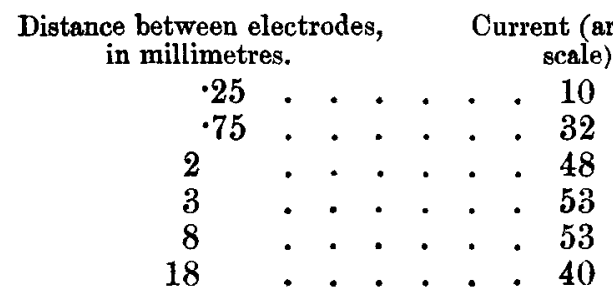

In this case the effect of distance is not so well marked as in the previous one, where the E.M.F. was sufficient to saturate the current at all distances.

The measurement of the rate of leak when the current is saturated enables us to form an estimate of the number of conducting particles present in the gas; as in this case the number of conducting particles produced in unit time by the rays is equal to the quantity of the electrolyte destroyed by the current in the same time. Let us take the case of hydrogen; when the current was saturated, the rate of leak between two plates each about $10 \mathrm{sq} . \mathrm{cm}$. in area and $1 \mathrm{~cm}$. apart was about 1 volt per second when a capacity of about $30 \mathrm{~cm}$. was in connexion with the electrometer. Thus the quantity of electricity passing between the plates in 1 second was 
about $10^{-1}$ electrostatic units, or $1 / 3 \times 10^{11}$ electromagnetic units, and this quantity is sufficient to electrolyse all the electrolytic gas produced by the Röntgen rays. Now 1 electromagnetic unit of electricity sets free $10^{-4}$ grammes of hydrogen, or about 1 c. c. at atmospheric temperature and pressure. Hence $1 / 3 \times 10^{11}$ electromagnetic units correspond to about the same number of cub. centim. of hydrogen ; the volume of the space between the electrodes was about 10 c.c., so in this experiment the fraction of the gas electrolysed was only $1 / 3 \times 10^{12}$, i.e., one three billionth of the whole amount of the gas. It is not surprising that some experiments we made to see if any alteration in pressure was produced when a gas was transmitting Röntgen rays should have given negative results. The preceding estimate gives the average number of conducting particles; if the conducting state is intermittent there may at certain times be a much larger number of these molecules present. It is probable that at all events, when the current is saturated the conducting power is intermittent. The action of the coil used to send the discharge through the vacuum tube is intermittent; thus, if between the passage of twe sparks the conductivity has time to vanish (and when any current is passing through the gas the rate at which it vanishes is very rapid) the gas will be alternately an insulator and then a conductor.

The following experiment is explained by the intermittent character of the discharge. The gas exposed to the Röntgen rays was in a piece of lead tubing open at both ends; this was connected with one terminal of a battery, the other terminal of which was connected with a wire running down the axis of the tube. A blast of air was blown through this tube, and it was found that when the current between the wire and the tube was small, the blast diminished the current to a large extent, though a current approaching saturation was hardly affected by the blast. When the current was affected the gas blown out of the tube was conducting; when the current was not affected the gas did not conduct. If the gas were exposed to steady radiation it would not be affected by blowing unless the time taken by the gas to acquire the conducting state under the influence of the rays was comparable with the time taken by the gas to pass through the tube; this is inconsistent with what we know from other experiments as to the rapidity of action of the rays. If, however, the state of the gas is intermittent, then, since the blast continues when the rays are not acting, it blows out conducting gas, and so diminishes its average conductivity.

To return to equation (3), if $I$ is the value of $\iota$ when $E$ 
398 Prof.J.J. Thomson and Mr. Rutherford on the Passu is infinite, we may write the equation in the form

$$
\mathrm{I}-\iota=\mathrm{C} \frac{\iota^{2}}{\mathrm{E}^{2}}, \text {. . . . . . }
$$

where

$$
\mathrm{C}=\frac{a l^{3}}{\epsilon \bar{U}^{2}},
$$

and is independent of both $\mathrm{E}$ and $\iota$

We have observed the relation between the current and the electromotive force for several gases, and for different intensities of the Röntgen rays. The comparison of the results of these experiments with equation (4) is given in the following tables :-

Leakage through Chlorine Gas.

$\begin{array}{ccc}\begin{array}{c}\text { Electromotive } \\ \text { Force. }\end{array} & \begin{array}{c}\text { Current } \\ \text { observed. }\end{array} & \begin{array}{c}\text { Current calculated } \\ \text { by equation 4. }\end{array} \\ * 9 & 65 & \\ 18 & 124 & 116 \\ 35 & 200 & 180 \\ * 70 & 245 & \\ 140 & 270 & 275\end{array}$

The observations marked with the asterisk were used to calculate the constants.

$\begin{array}{rcr} & \text { Leakage through Air. } \\ * 9 & 22 & \\ 18 & 39 & 38 \\ 35 & 67 & 67 \\ * 70 & 83 & \\ 140 & 90 & 86\end{array}$

The observations marked with the asterisk were used to calculate the value of the constants in equation 4 .

Leakage through Hydrogen.

$\begin{array}{rrr}5 & 18 & 19 \\ * 9 & 31 & \\ 18 & 53 & 48 \\ 35 & 63 & 58 \\ * 70 & 65 & \end{array}$

The observations marked with the asterisks were used to calculate the constants in equation 4 . 
of Electricity through Gases exposed to Röntgen Rays. 399

Leakage through Chlorine. (Strong radiation.)

$\begin{array}{ccc}\begin{array}{c}\text { Electromotive } \\ \text { Force. }\end{array} & \begin{array}{c}\text { Current } \\ \text { observed. }\end{array} & \begin{array}{c}\text { Current oalc } \\ \text { by equation }\end{array} \\ 5 & 53 & 53 \cdot 4 \\ * 10 & 100 & \\ 21 & 189 & 183 \\ 35 & 275 & 255 \\ * 70 & 355 & \\ 140 & 380 & 405\end{array}$

Leakage through Chlorine. (Weak radiation.)

$\begin{array}{ccc}* 5 & 10 & \\ 8 \cdot 5 & 16 & 15 \\ 17 & 26 & 23 \\ * 35 & 32 & \\ 105 & 34 & 37\end{array}$

Coal Gas (1).

$\begin{array}{rll}1 \cdot 4 & 10 & 9 \cdot 8 \\ 2 \cdot 8 & 17 \cdot 3 & \\ 4 \cdot 2 & 22 & 23 \\ 8 \cdot 4 & 32 \cdot 3 & 33 \\ 16 \cdot 8 & 38 \cdot 3 & 40 \\ 35 & 43 & \\ 110 & 45 & 44\end{array}$

Coal Gas (2). Weak radiation.

$\begin{array}{rlr}1 \cdot 4 & 3 \cdot 6 & 4 \cdot 2 \\ 2 \cdot 8 & 8 & \\ 4 \cdot 2 & 11 & 11 \cdot 2 \\ 5 \cdot 6 & 14 \cdot 7 & 15 \cdot 2 \\ 8 \cdot 4 & 21 \cdot 7 & 21 \cdot 9 \\ 12 \cdot 6 & 32 & 30 \cdot 4 \\ 16 \cdot 8 & 38 & \end{array}$

Hydrogen.

$\begin{array}{rlr}3 \cdot 4 & 5 & \\ 5 \cdot 1 & 7 \cdot 5 & 6 \cdot 9 \\ 8 \cdot 5 & 10 & 10 \cdot 1 \\ 15 \cdot 6 & 15 & 13 \cdot 4 \\ 34 & 16 \cdot 6 & \\ 68 & 16 \cdot 6 & 17\end{array}$


400 Prof. J. J. Thomson and Mr. Rutherford on the Passage

\begin{tabular}{|c|c|c|}
\hline \multicolumn{3}{|c|}{ Sulphuretted Hydrogen. (Strong radiation.) } \\
\hline $\begin{array}{l}\text { Electromotive } \\
\text { Foree. }\end{array}$ & $\begin{array}{l}\text { Current } \\
\text { observed. }\end{array}$ & $\begin{array}{c}\text { Current calculated } \\
\text { by equation } 4 .\end{array}$ \\
\hline $\begin{array}{l}15 \cdot 6 \\
34 \\
68\end{array}$ & $\begin{array}{c}8 \cdot 7 \\
18 \\
30 \cdot 8\end{array}$ & $\begin{array}{l}17 \cdot 1 \\
28 \cdot 5\end{array}$ \\
\hline 126 & & \\
\hline
\end{tabular}

Sulphuretted Hydrogen. (Weak radiation.)

$\begin{array}{rll}15 \cdot 6 & 3 \cdot 8 & \\ 34 & 6 \cdot 3 & 6 \cdot 2 \\ 68 & 8 & 8 \\ 136 & 8 \cdot 7 & \\ & \text { Mercury Vapour. } \\ 5 \cdot 1 & 14 \cdot 2 & 14 \cdot 6 \\ 8 \cdot 5 & 23 & \\ 15 \cdot 6 & 35 & 36 \cdot 9 \\ 34 & 55 & 59 \\ 68 & 75 & \\ 136 & 75 & 8 \cdot 2\end{array}$

As these measurements require the intensity of the radiation to be maintained constant during each series of ohservations, a condition which it is very difficult to fulfil, we think the agreement between theory and observation is as close as could be expected.

We have seen how from the measurement of the limiting current we could form an estimate of the proportion which the conducting particles bear to the rest of the molecules of the gas. We can, in addition, get from the curve representing the relation between the current and the electromotive force an estimate of the velocity with which these particles move. Taking equation (3)

$$
q-\frac{\alpha l^{2} \iota^{2}}{\epsilon^{2} \mathrm{U}^{2} \mathrm{E}^{2}}-\frac{\iota}{l \epsilon}=0,
$$

we shall endeavour to express the coefficients in terms of quantities which our experiments enable us to estimate. Let I be the limiting current when the electromctive force is infinite, then

$$
\mathrm{I}=q l \epsilon .
$$

Let $\mathrm{T}$ be the time which elapses after the rays have been stopped for the number of conducting particles to fall to one half the number just before the rays ceased, no current passing through the gas. Then, just before the rays cease to fall on 
of Electricity through Gases exposed to Röntgen Rays. 401 the gas, we have from equation (2),

$$
\mathrm{N}=\left\{\frac{q}{\alpha}\right\}^{\frac{1}{2}}
$$

where $\mathrm{N}$ represents the number of conducting particles at this stage; after the rays have ceased, we have

$$
\frac{d n}{d t}=-\alpha n^{2}
$$

or

$$
\frac{1}{n}-\frac{1}{\mathrm{~N}}=a t,
$$

if $t$ is the time which has elapsed after the rays have stopped, when $t=\mathrm{T}, n=\frac{1}{2} \mathrm{~N}$, hence

$$
\frac{1}{\mathrm{~N}}=\alpha \mathrm{T}
$$

substituting for $\mathrm{N}$ its value, we get

or

$$
\begin{gathered}
\mathrm{T}^{2}=\frac{1}{\alpha q}, \\
\alpha=\frac{1}{\mathrm{~T}^{2} q}=\frac{l \epsilon}{\mathrm{T}^{2} \mathrm{I}} .
\end{gathered}
$$

Substituting for $q$ and $a$ the values just found, equation (4) becomes

or

$$
\frac{\mathrm{I}-\iota}{l \epsilon}=\frac{l^{8} \iota^{2} \epsilon}{\mathrm{IT}^{2} \epsilon^{2} \mathrm{E}^{2} \mathrm{U}^{2}}
$$

$$
\mathrm{I}(\mathrm{I}-\iota)=\frac{l^{4} \iota^{2}}{\mathrm{~T}^{2} \mathrm{E}^{2} \mathrm{U}^{2}} \cdot \quad . \quad \cdot \quad \cdot \quad \cdot
$$

Thus in the straight part of the curve, where $\iota$ is small compared with I, we have approximately

$$
\frac{\iota}{\mathrm{l}}=\frac{\mathrm{EUT}}{l^{2}} \ldots . \quad \cdot \quad \cdot \quad \cdot \quad \cdot
$$

Now EU/l is the sum of the velocities of the positively and negatively charged particles in the electric field. Hence, equation (6) shows that the current bears to the maximum current the same ratio as the space described by the charged particles in time $T$ bears to the distance between the electrodes. In an experiment where $l$ was about $1 \mathrm{~cm}$., the rate of leak through air for a potential-difference of 1 volt was about $\frac{1}{30}$ of the maximum rate of leak, hence the charged 


\section{Prof. J.J. Thomson and Mr. Rutherford on the Passage}

particles must in the time $T$ have moved through about $\frac{1}{30}$ of a centimetre. The time $\mathbf{T}$ will depend upon the intensity of the radiation; it could be determined by measuring the rate of leak at different points on the tube through which the conducting gas was blown in the experiment mentioned at the beginning of this paper. We hope to make such experiments and obtain exact values for $\mathrm{T}$; in the meantime, from the rough experiments already made, we think we may conclude that with the intensity of radiation we generally employed, $T$ was of the order of $\frac{1}{10}$ of a second. This would make the velocities of the charged particles in the air about $.33 \mathrm{~cm}$. $/ \mathrm{sec}$. for a gradient of one volt per $\mathrm{cm}$. This velocity is very large compared with the velocity of ions through an eleetrolyte ; it is, however, small compared with the velocity with which an atom carrying an atomic charge would move throngh a gas at atmospheric pressure; if we calculate by tlie kinetic thsory of gases this velocity, we find that for air it is of the order $50 \mathrm{~cm}$. $/ \mathrm{sec}$.; this result seems to imply that the charged particles in the gas exposed to the Röntgen rays are the centres of an aggregation of a considerable number of molecules.

The relation between the current and electromotive force given by equation (4) corresponds to that obtained by experiment for a number of gases; it does not, however, exhibit a peculiarity which we have sometimes observed, especially when the radiation was strong, $i$. e., the existence of a part of the curve where the current increases faster than would be the case if Obm's law were true; this is shown by the portion $\mathrm{EF}$ of the curve in fig. 2, which represents the relation be-

Fig. 2.

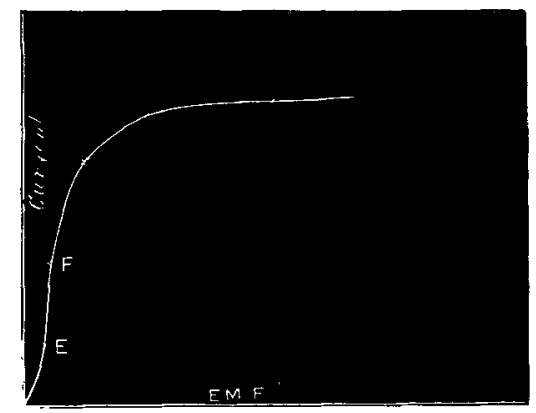

tween the current and electromotive force through sulphuretted hydrogen. When the intensity of the Röntgen rays is altered, the alteration in the current is not the same 
of Electricity through Gases exposed to Röntgen Rays. 403

at different points in the curve. When the intensity of these rays is diminished, the saturation current is diminished in a larger proportion than the current for small electromotive forces. This is shown by the following diagram, which represents the $\iota$ and E curres through chlorine gas for different intensities of the Röntgen rays; the weak radiation was got by interposing a thick aluminium plate. In this diagram

Fig. 3.

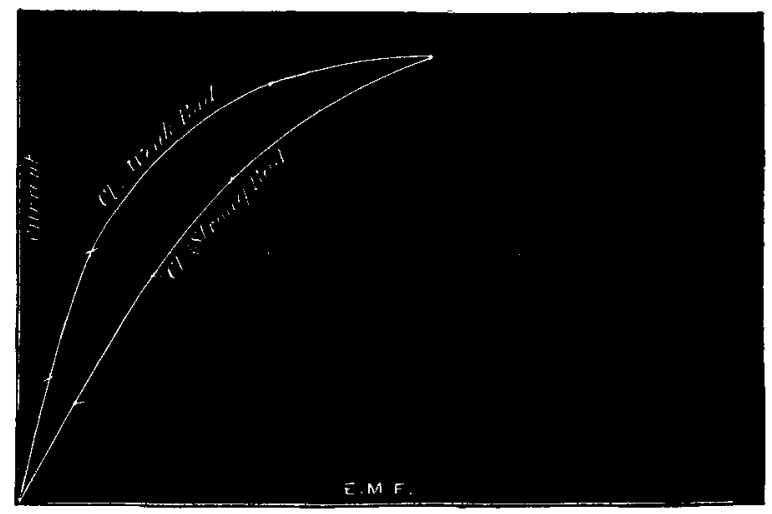

the ordinates for the weak radiation have been increased so as to make the ordinate for the saturation current of the weak radiation the same as that of the strong. When this is done the rest of the "weak" curve is above the strong, showing that the diminution in the radiation has affected the saturation current to a greater extent than the weaker currents. The saturation current depends only on the number of conducting particles produced by the rays ; for the smaller currents the diminution in the number of molecules is to some extent compensated for by the increase in the time taken for these to recombine; thus $\mathrm{T}$ is increased when the intensity of the rays is diminished, so that, as we see from equation (6), the proportion between a small current and the saturation current is increased when the intensity of the rays is diminished.

Whatever is the magnitude of the electromotive force, a dininution in the intensity of the rays is accompanied by a diminution in the current, so that the $I$ and $E$ curves for two intensities of radiation would not intersect if both were dran $n$ on the same scale.

If, however, instead of keeping the gas the same and altering the intensity of the radiation, we alter the gas and 
404 Prof. J. J. Thomson and Mr. Rutherford on the Passage keep the intensity of the rays constant, then the $\mathbf{I}$ and $\mathbf{E}$ curves for two different gases may intersect. This effect is shown in the following diagram, which represents the I and $\mathbf{E}$ curves for hydrogen and air. We see that for small electromotive forces the current is greater in hydrogen than in air, while the saturation current is much greater in air than in hydrogen. The saturation current depends merely

Fig. 4.

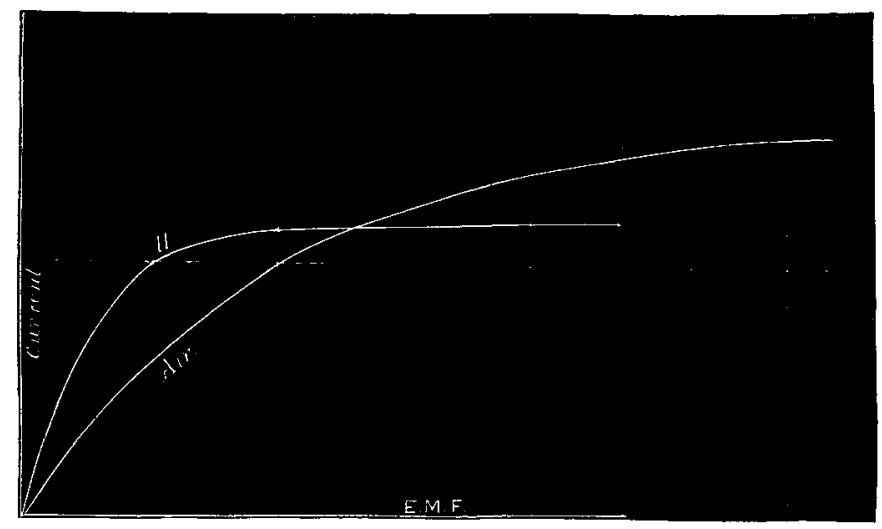

on the number of conducting particles produced by the rays, while the current in the earlier part of the curve depends on the space described by the conducting particles in the time $T$ (see equation 6), and we infer that more conducting particles are produced by the rays in air than in hydrogen, but that the product of $U$, the velocity of these particles, and 'T, a time which is proportional to the time these particles linger after the rays are cut off, is greater for hydrogen than it is for air.

In fig. 5 we give the curves for air, chlorine, sulphuretted hydrogen, and mercury vapour, the eurves being drawn on such scales that the ordinate representing the saturation current is the same in all these cases. It will be noticed that the curves for air, for sulphuretted hydrogen, and for chlorine coincide, mercury vapour falls below, while the bydrogencurve would be above. This shows that, using the notation of equation (6), UT is the same for air, chlorine, and sulphuretted bydrogen, and that its value for these gases is smaller than for hydrogen and greater than for mercury vapour.

It is remarkable that the shapes of the curves for air, sulphuretted hydrogen, and chlorine should agree so closely, 
of Electricity through Gases exposed to Röntgen Rays. 405

for the absolute values of the current in these gases is very different, the saturation current in sulphuretted hydrogen

Fig. 5.

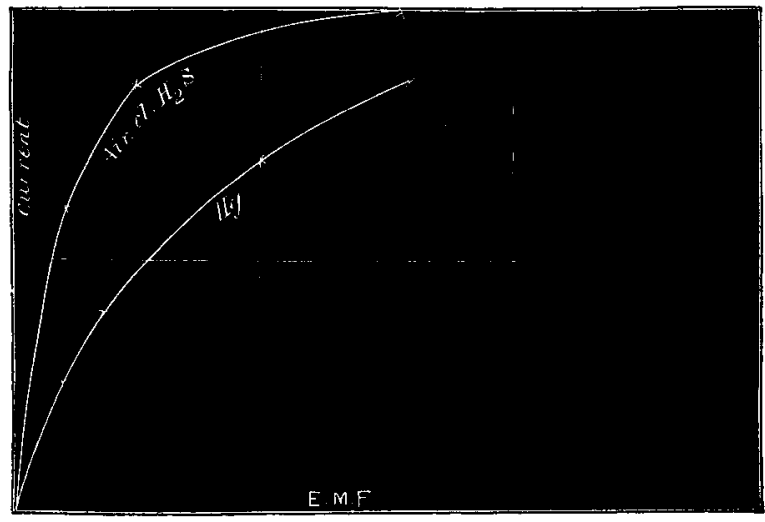

being in some cases three or four times that of air, while that of chlorine is in some cases as much as ten times that of air.

The value of the saturation current varies greatly in different gases; of the gases we have tried it is least in hydrogen, greatest in mercury vapour, the saturation current in mercury vapour being about 20 times that for air. It does not seem to depend entirely on the density of the gas, as in sulphuretted hydrogen it is three or four times what it is in air, though the densities are nearly equal, while, though the density of the vapour of $\mathrm{CH}_{2} \mathrm{I}_{2}$ is greater than that of mercury vapour, the saturation current in the former gas is only a small fraction of its value for the latter. The gases which have large saturation currents are those which contain the elements which have an abnormally large specific inductive capacity in comparison with their valency.

We have made a large number of experiments with the view to seeing whether there is any polarization when a current of electricity passes through the gas; we have not, however, been able to satisfy ourselves of the existence of this effect. The absence of polarization implies, however, that the ions are able to give up their charges to the metal electrodes. Experiments on electritied gases show, however, that it is very difficult to get a charge of electricity from a gas to a metal unless the metal is exposed to radiation, either by the metal being sufficiently hot to be lnminous, or when it is exposed to ultra-violet light. But in the case of the passage of electricity through a gas which has been expossed to Röntgen rays the Phil. Mag. S. 5. Vol. 42. No. 258. Nov. 1896. $2 \mathrm{G}$ 
conduction takes place even when the system is not exposed to the direct radiation fron the exhausted tube; we think it probable therefore that the gas itself radiates after being exposed to the Röntgen rays.

To test this we tried the following experiment. AB, CD Fig. 6.

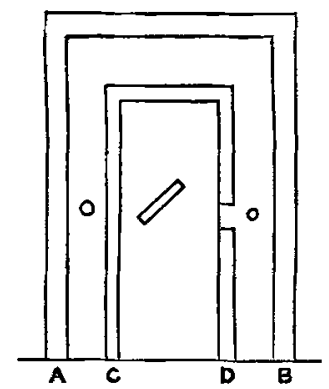

are two concentric cylinders made of thick lead tubing, the base of the inner one was cardboard, so as to allow Röntgen rays to pass through the gas in the inner cylinder. A metal ring was placed between the two cylinders and connected with one pair of quadrants of an electrometer so as to allow the leak from it when raised to a high potential to be measured. A slit was cut in the inner cylinder in such a place and of such a size that no rays could pass through it directly from the bulb. The apparatus was filled with chlorine, as this gas is one which gives a very rapid rate of leak. When the slit was left open there was a rapid leak due to the diffusion from the inner cylinder of gas which had been exposed to Röntgen rays. When, however, the slit was covered up with a strip of payer the leak wholly disappeared, though the ring connected with the electrometer was placed at the same level as the slit and therefore exposed to any radiation that might come from the gas. 'This radiation, if it exists, must therefore either be of very feeble intensity or else it must differ from the Röntgen rays in not making a gas through which it passes a conductor of electricity. We are inclined to think that when Röntgen rays are incident on a metallic surface the "diffisely reflected" rays are not of the same character as the incident ones, and have not nearly the same power of rendering a gas through which they pass a conductor of electricity. We base this opinion on the experiments we have made to detect the existence of electrical effects due to the "reflected" rays; though we have made many attempts we have never been able to detect the existence of any electrical 
effects from the reflected rays. Thus we introduced in the apparatus in fig. 6 a lead plate inclined at an angle of $45^{\circ}$ to the axis of the cylinder, and so placed as to reflect the rays through the slit, which was covered with a strip of paper; the arrangement was so sensitive that if the plate had reflected anything like one per cent. of the rays incident upon it, the leak from the metal ring would have been easily detected; there was, however, no trace of a leak. The results of experiments on the photographic effects produced by ray's diffusely reflected from metallic plates seem to show that these rays are fairly abundant. Taking this result in connexion with the absence of any noticeable electrical effect produced by these diffusely reflected rays, we think that the latter differ in character from the incident rays.

We have not been able to detect any effect produced by a magnetic field on the rate of leak ; we tried with the lines of magnetic force parallel and also at right angles to the current, and with both small and saturated currents.

The rate of leak through air that had been dried by standing for three days in the presence of phosphorus pentoxide did not differ appreciably from the damp air of the room.

In conclusion, we desire to thank Mr. E. Everett for the assistance he has given us in these experiments. The period during which a bulb gives ont Röntgen rays at a uniform rate is not a long one, and as most of our experiments required the rate of emission to be constant, they have entailed the use of a very large number of bulbs, all of which have been made by Mr. Everett.

XLI. On the Resistance of the Electric Arc. By JuLIus Frith, 1851 Exhibition Scholar, the Owens College, Manchester, and Charless Rodgers, B.Sc., 18.51 Exhibition Scholar, Firth College, Sheffield*.

\section{[Plates III. to V.]}

THERE seems to be some uncertainty as to what is meant 1 by the resistance of the are. Any given are is a phenomenon which exists at a detinite P.D. and current, and any attempt to measure its resistance must alter the state of the arc as little as possible or else we are no longer dealing with the same phenomenon. Hence it seems to us that the only way in which the resistance of the arc can be measured is by the ratio of a very small increment of P.D. applied, to

* Communicated by the Physical Societv : read May 8, 1896. 